\title{
Acceleration of Electron-Positron Plasmas to High Energies
}

\author{
ABRAHAM LOEB, LAZAR FRIEDLAND, AND SHALOM ELIEZER
}

\begin{abstract}
It is suggested that electron-positron $\left(\mathrm{e}^{+} \mathrm{e}^{-}\right)$plasma can be accelerated using the concept of cyclotron autoresonance between the particles and a linearly polarized laser radiation propagating along an axial magnetic field $\left(B_{z}\right)$. This scheme can also be applied for other plasmas with oppositely charged particles of equal $|q| / m$ (e.g., positive and negative ions). An $\mathrm{e}^{+} \mathrm{e}^{-}$plasma can be accelerated to about 2 $\mathrm{GeV}$ in the first meter along a 100-kG guide magnetic field by using an Nd: glass laser $\left(\lambda_{0}=1 \mu \mathrm{m}\right)$ with intensity $I_{0}=10^{18} \mathrm{~W} / \mathrm{cm}^{2}$. The acceleration scales asymptotically as $\left(B_{z} I_{0} \lambda_{0} z^{2}\right)^{1 / 3}$, where $z$ is the axial distance.
\end{abstract}

\section{INTRODUCTION}

A CCELERATION of particles to high energies by high-power lasers has received considerable attention in recent years [1]-[3]. Several schemes for coupling between the laser field and relativistic charged beams have been proposed. Two major schemes applying the far-field approach were discussed: the inverse free-electron laser (IFEL) [4] and recently the autoresonance laser accelerator (ALA) [5]. ${ }^{1}$ In the IFEL accelerator the attainable energies are limited ( $\$ 400 \mathrm{GeV}$ ) due to radiation losses, and the accelerated beam current is limited as a result of transverse gradients of the wiggler field.

The ALA scheme is based on a cyclotron autoresonance between charged particles and an electromagnetic wave propagating along an axial guide magnetic field. This selfsustained resonance is achieved for luminous radiation and homogeneous fields. Both the nonlinear single particle [5] and the collective [6] behavior of this system have been analyzed recently for the case of a circularly polarized laser radiation. It has been shown that the accelerated beam can be launched into the desired autoresonance regime through an appropriate transition region. The possibility of using the megagauss magnetic fields generated spontaneously in laser-produced plasmas for autoreso-

Manuscript received September 1, 1986; revised November 15, 1986. A. Loeb is with the Center for Plasma Physics, Racah Institute of Physics, Hebrew University of Jerusalem, 91904 Jerusalem, Israel, and with the Plasma Department, Soreq Nuclear Research Center, 70600 Yavne, Israel.

L. Friedland is with the Center for Plasma Physics, Racah Institute of Physics, Hebrew University of Jerusalem, 91904 Jerusalem, Israel. He is now on sabbatical with the Lawrence Berkeley Laboratory, Berkeley, CA 94720 .

S. Eliezer is with the Plasma Department, Soreq Nuclear Research Center, 70600 Yavne, Israel.

IEEE Log Number 8613323.

${ }^{1}$ The autoresonance accelerator term was also used by Sloan and Drummond (see Phys. Rev. Lett, vol. 31, p. 1234, 1973) to describe a collective ion acceleration scheme, different from the ALA. nance acceleration was also suggested [7]. The ALA concept allows the acceleration of high-current beams to teraelectronvolt energies with low radiation losses. However, generally speaking, the collective acceleration of dense beams is limited by space-charge effects. In this paper we propose a novel approach to cancel these effects by accelerating "quasi-neutral" beams (plasmas) to high energies applying the ALA concept. In particular, we consider the simultaneous acceleration of guided electrons and positrons by a linearly polarized electromagnetic radiation. Lately, the autoresonance acceleration of pairs by a nonpropagating electromagnetic wave has been discussed [8]. However, this approach cannot be extended to high energies because of synchrotron radiation losses.

The scope of the present work is as follows. The nonlinear electron dynamics in combined guide magnetostatic and linearly polarized laser radiation fields is considered analytically in Section II for homogeneous fields and luminous radiation. It is shown that at these conditions two autoresonances can be achieved. In Section III we analyze the symmetry between the autoresonant acceleration of a positron and an electron. This symmetry allows the simultaneous acceleration of electron-positron pairs to high energies. Finally, Section IV summarizes the results of this work.

\section{The Electron Dynamics in Combined Guide Magnetostatic Field and Linearly Polarized ElECTROMAGNETIC RADIATION}

Consider a plane electromagnetic wave propagating in the $z$ direction along an axial homogeneous magnetostatic field, $\vec{B}_{z}$. Let $\vec{k}_{0}$, $\omega_{0}^{\prime}$ be the wave vector and the frequency of the wave, respectively. The following two sets of orthonormal vectors are defined:

$$
\begin{aligned}
& \left\{\begin{array}{l}
\hat{e}_{1}=-\hat{e}_{x} \sin \phi+\hat{e}_{y} \cos \phi \\
\hat{e}_{2}=-\hat{e}_{x} \cos \phi-\hat{e}_{y} \sin \phi \\
\hat{e}_{3}=\hat{e}_{z}
\end{array}\right\} \\
& \left\{\begin{array}{l}
\hat{e}_{1}=-\hat{e}_{x} \sin \phi-\hat{e}_{y} \cos \phi \\
\hat{e}_{2}=-\hat{e}_{x} \cos \phi+\hat{e}_{y} \sin \phi \\
\hat{e}_{3}=\hat{e}_{z}
\end{array}\right\}
\end{aligned}
$$


where, $\phi=k_{0} z-\omega_{0}^{\prime} t$. The pairs of vectors $\left\{\hat{e}_{1}, \hat{e}_{2}\right\}$ and $\left\{\hat{\tilde{e}}_{1}, \hat{\tilde{e}}_{2}\right\}$ are rotating in opposite directions as functions of $\phi$. The transformation between the two sets of vectors is

$$
\begin{aligned}
& \hat{\tilde{e}}_{1}=-\cos (2 \phi) \hat{e}_{1}+\sin (2 \phi) \hat{e}_{2} \\
& \hat{\tilde{e}}_{2}=\sin (2 \phi) \hat{e}_{1}+\cos (2 \phi) \hat{e}_{2} \\
& \hat{\tilde{e}}_{3}=\hat{e}_{3} .
\end{aligned}
$$

The electromagnetic fields in the systems can be written as

$$
\begin{aligned}
\vec{E} & =-\frac{1}{c} \frac{\partial \vec{A}}{\partial t} \\
\vec{B} & =\vec{\nabla} \times \vec{A}+B_{z} \hat{e}_{3}
\end{aligned}
$$

where the vector potential is assumed to be linearly polarized in the $x$ direction:

$$
\vec{A}=A_{x} \cos \phi \hat{e}_{x}=-A \hat{e}_{2}-A \hat{\tilde{e}}_{2}
$$

and

$$
A=A_{x} / 2 .
$$

Thus the electromagnetic wave can be decomposed into right- and left-handed circularly polarized waves. The amplitude of each of these waves is half of the amplitude of the linearly polarized wave.

We shall discuss in the following the dynamics of a cold electron beam in the described fields configuration, assuming that $\vec{E}$ and $\vec{B}$ are large enough and thus are not affected by the beam itself. The electron's momentum equation is

$$
\left(\frac{\partial}{\partial t}+\vec{v} \cdot \vec{\nabla}\right)(m \gamma \vec{v})=-e\left(\vec{E}+\frac{\vec{v}}{c} \times \vec{B}\right)
$$

where $\gamma=\left(1-v^{2} / c^{2}\right)^{-1 / 2}$.

Equivalently, from (3),

$$
\left(\frac{\partial}{\partial t}+\vec{u} \cdot \vec{\nabla}\right)(\gamma \vec{u}-\vec{\alpha})=\vec{\Omega} \times \vec{u}-(\vec{\nabla} \vec{\alpha}) \cdot \vec{u}
$$

where we use the following notations:

$$
\begin{aligned}
\vec{\Omega} & =\left(e B_{z} / m c^{2}\right) \hat{e}_{3} \\
\vec{\alpha} & =e \vec{A} / m c^{2} \\
\vec{u} & =\vec{v} / c \\
\omega_{0} & =\omega_{0}^{\prime} / c \\
\tau & =c t .
\end{aligned}
$$

According to (2) and (4),

$$
\vec{\alpha}=\alpha_{x} \cos \phi \hat{e}_{x}=-\alpha \hat{e}_{2}-\alpha \hat{\tilde{e}}_{2}=\alpha_{1} \hat{e}_{1}+\alpha_{2} \hat{e}_{2}
$$

where $\alpha_{1}=-\alpha \sin (2 \phi), \alpha_{2}=-2 \alpha \cos ^{2} \phi$, and $\alpha=$ $e A_{x} / 2 m c^{2}=\alpha_{x} / 2$. Next we write (6) in components in the frame $\left\{\hat{e}_{j}\right\}$ :

$\frac{d}{d \tau}\left(\gamma u_{1}-\alpha_{1}\right)+\left(\omega_{0}-k_{0} u_{3}\right)\left(\gamma u_{2}-\alpha_{2}\right)=-\Omega u_{2}$

$$
\begin{gathered}
\frac{d}{d \tau}\left(\gamma u_{2}-\alpha_{2}\right)-\left(\omega_{0}-k_{0} u_{3}\right)\left(\gamma u_{1}-\alpha_{1}\right)=\Omega u_{1} \\
\frac{d}{d \tau}\left(\gamma u_{3}\right)=k_{0}\left(\alpha_{2} u_{1}-\alpha_{1} u_{2}\right) .
\end{gathered}
$$

The energy of the electron evolves according to the equation

$$
\frac{d \gamma}{d \tau}=u_{1} \frac{\partial \alpha_{1}}{\partial \tau}+u_{2} \frac{\partial \alpha_{2}}{\partial \tau}+\omega_{0}\left(\alpha_{2} u_{1}-\alpha_{1} u_{2}\right) .
$$

For homogeneous and stationary fields ( $\alpha, \Omega=$ constant) (8)-(11) can be simplified by using (7):

$$
\begin{aligned}
& \left(\gamma u_{1}\right)^{\cdot}=k_{0}\left(u_{3}-u_{p}\right)\left(\gamma u_{2}+2 \alpha \sin ^{2} \phi\right)-\Omega u_{2} \\
& \left(\gamma u_{2}\right)^{\cdot}=-k_{0}\left(u_{3}-u_{p}\right)\left(\gamma u_{1}-\alpha \sin (2 \phi)\right)+\Omega u_{1} \\
& \left(\gamma u_{3}\right)^{\cdot}=\left(\dot{\gamma} / u_{p}\right)=-2 k_{0} \alpha\left(u_{1} \sin \phi+u_{2} \cos \phi\right) \sin \phi
\end{aligned}
$$

where $(\cdots)^{\cdot} \equiv d(\cdots) / d \tau$. These are the desired equations describing the electron dynamics in the system. Equation (14) yields the following constant of motion:

$$
L_{0}=\gamma\left(1-u_{p} u_{3}\right)=\text { constant } .
$$

Note that (12)-(14) include expressions containing $\phi$. Therefore, their direct time integration is difficult since $\phi$ is a function of $z$ and

$$
z(\tau)=\int_{0}^{\tau} u_{3} d \tau
$$

One can overcome this difficulty by replacing the time derivatives in these equations by phase derivatives. For an arbitrary variable $a$, we have

$$
(a)^{\cdot}=\dot{\phi}(d a / d \phi) \equiv \dot{\phi}(a)_{\phi}=k_{0}\left(u_{3}-u_{p}\right)(a)_{\phi} \text {. }
$$

Therefore, (12) and (13) can be rewritten as

$$
\begin{aligned}
& \left(\gamma u_{1}\right)_{\phi}=l\left(\gamma u_{2}\right)+2 \alpha \sin ^{2} \phi \\
& \left(\gamma u_{2}\right)_{\phi}=-l\left(\gamma u_{1}\right)+\alpha \sin (2 \phi)
\end{aligned}
$$

where $l=1+\Omega /\left[k_{0} \gamma\left(u_{p}-u_{3}\right)\right]$. Equations (18) and (19) exhibit simple resonance behavior when $l=$ constant. According to (15), this takes place only for luminous radiation, i.e., when $u_{p}=1$. We will consider the electron dynamics at this case in the following. Equations (18) and (19) yield a "forced harmonic oscillator" type of differential equation for $\left(\gamma u_{1}\right)$ :

$$
\left(\gamma u_{1}\right)_{\phi \phi}+l^{2}\left(\gamma u_{1}\right)=(2+l) \alpha \sin (2 \phi)
$$

where $l=1+\Omega /\left(k_{0} L_{0}\right)$. The last equation shows the existence of two self-sustained cyclotron resonances in the system, namely,

$$
\begin{aligned}
& l=0<=>\Omega=-k_{0} L_{0} \\
& l=2<=>\Omega=k_{0} L_{0} .
\end{aligned}
$$


The two autoresonances differ in the direction of the magnetic field. According to (15),

$$
u_{3}=1-L_{0} / \gamma
$$

and since $\gamma=\left(1-u_{1}^{2}-u_{2}^{2}-u_{3}^{2}\right)^{-1 / 2}$, we obtain

$$
\gamma=\frac{1}{2 L_{0}}\left[1+\left(\gamma u_{1}\right)^{2}+\left(\gamma u_{2}\right)^{2}+L_{0}^{2}\right]
$$

Thus it is evident that

$$
\lim _{|\phi| \rightarrow \infty} \gamma \rightarrow \infty
$$

if

$$
\lim _{|\phi| \rightarrow \infty}\left(\gamma u_{1}\right) \rightarrow \infty \text {. }
$$

First, we consider the electron dynamics at the autoresonance: $l=0$. In this case the solutions of (18) and (19) are

$$
\begin{aligned}
& \gamma u_{1}=\gamma_{0} u_{10}+\alpha\left(\phi-\frac{1}{2} \sin (2 \phi)\right) \\
& \gamma u_{2}=\gamma_{0} u_{20}+\alpha \sin ^{2} \phi .
\end{aligned}
$$

In order to find the time dependence of the electron's energy we use the equalities

$$
\gamma \dot{\gamma}=k_{0} \gamma\left(u_{3}-1\right) \gamma_{\phi}=-k_{0} L_{0} \gamma_{\phi}
$$

and, from (14) and (24)-(26)

$$
\begin{aligned}
\gamma_{\phi}=(2 \alpha & \left.\sin \phi / L_{0}\right)\left[\gamma _ { 0 } \left(u_{10} \sin \phi\right.\right. \\
& \left.\left.+u_{20} \cos \phi\right)+\alpha \phi \sin \phi\right] .
\end{aligned}
$$

The integration of the last equation yields

$$
\begin{aligned}
\gamma= & \gamma_{0}+\left(\alpha / 2 L_{0}\right)\left[-\gamma_{0}\left(u_{10} \sin (2 \phi)+u_{20} \cos (2 \phi)\right)\right. \\
& +2 \gamma_{0} u_{10} \phi \\
& \left.+\gamma_{0} u_{20}+\alpha\left(\phi^{2}-\phi \sin (2 \phi)+\sin ^{2} \phi\right)\right] .
\end{aligned}
$$

The time dependence of $\phi$ for $l=0$ can be found from

$$
\dot{\phi}=k_{0}\left(u_{3}-1\right)=\Omega / \gamma
$$

yielding

$$
\tau=\frac{1}{\Omega} \int \gamma \dot{\phi} d \tau=\frac{1}{\Omega} \int \gamma(\phi) d \phi
$$

By substituting (28) into (30) we have

$$
\begin{aligned}
\tau= & \frac{\gamma_{0}}{\Omega} \phi+\left(\frac{k_{0} \alpha}{2 \Omega^{2}}\right)\left[\frac{\gamma_{0}}{2}\left(u_{10} \cos (2 \phi)-u_{20} \sin (2 \phi)\right)\right. \\
& -\gamma_{0} u_{10} \phi^{2} \\
& -\gamma_{0} u_{20} \phi-\frac{1}{2} \gamma_{0} u_{10}-\alpha\left(\frac{\phi^{3}}{3}+\frac{1}{2} \phi(\cos (2 \phi)\right. \\
& \left.\left.+1)-\frac{1}{2} \sin (2 \phi)\right)\right] .
\end{aligned}
$$

Equations (22), (24), (25), (28), and (31) describe the nonlinear dynamics of the electron at the resonance $l=$ 0 . Asymptotically, for $\phi \rightarrow-\infty$, one obtains

$$
\begin{aligned}
\vec{u}_{\perp} & \simeq u_{1} \hat{e}_{1} \simeq \frac{\alpha \phi}{\gamma} \hat{e}_{1} \simeq-\sqrt{\frac{2 L_{0}}{\gamma}} \hat{e}_{1} \\
\phi & \simeq-\left(\frac{6 \Omega^{2}}{k_{0} \alpha^{2}} \tau\right)^{1 / 3}=-7.22\left(\left(\frac{B_{z}(100 \mathrm{kG})}{\alpha}\right)^{2}\right. \\
\gamma & \left.\simeq\left(\frac{3|\alpha|}{\sqrt{2}}\left(k_{0}|\Omega|\right)^{1 / 2} \tau\right)^{2 / 3}\right)^{1 / 3} \\
& =2.6 \cdot 10^{3} \cdot\left(|\alpha|\left|\frac{B_{z}(100 \mathrm{kG})}{\lambda_{0}(10 \mu \mathrm{m})}\right|^{1 / 2} z(m)\right)^{2 / 3}
\end{aligned}
$$

where $\lambda_{0}=\left(2 \pi / k_{0}\right)$, is the radiation wavelength. These asymptotic results coincide with the results obtained previously [5] for circularly polarized radiation: $\vec{\alpha}=\alpha_{2} \hat{e}_{2}$, if we perform the transformation: $-\alpha=-\left(\alpha_{x} / 2\right) \rightarrow \alpha_{2}$. At the resonance $l=0$ the electron is accelerated by the circularly polarized component: $-\alpha \hat{e}_{2}$ of $\vec{\alpha}$ (see (7)). Therefore, the accelerating amplitude is only one-half of the amplitude of the linearly polarized radiation.

Next, we consider the autoresonance $l=2$. For this case, (20) has the solution

$$
\begin{aligned}
\gamma u_{1}= & \left(\gamma_{0} u_{20}+\alpha / 2\right) \sin (2 \phi) \\
& +\left(\gamma_{0} u_{10}-\alpha \phi\right) \cos (2 \phi)
\end{aligned}
$$

and using (19) we get

$\gamma u_{2}=\gamma_{0} u_{20} \cos (2 \phi)-\left(\gamma_{0} u_{10}-\alpha \phi\right) \cos (2 \phi)$.

Therefore, for $l=2$ and $u_{1}(\phi=0)=u_{10}$ the electron transverse momentum components in the frame $\left\{\hat{\hat{e}}_{j}\right\}$ are identical with those for $l=0$ and $u_{1}(\phi=0)=-u_{10}$ in the frame $\left\{\hat{e}_{j}\right\}$. Furthermore, (14), (35), and (36) yield

$$
\begin{aligned}
\gamma_{\phi}= & \left(2 \alpha \sin \phi / L_{0}\right)\left[\gamma _ { 0 } \left(u_{20} \cos \phi\right.\right. \\
& \left.\left.-u_{10} \sin \phi\right)+\alpha \phi \sin \phi\right] .
\end{aligned}
$$

Thus from (27) and (37) we conclude that the autoresonance acceleration at $l=0$ and $u_{1}(\phi=0)=u_{10}$ is the same as the autoresonance acceleration at $l=2$ and $u_{1}(\phi$ $=0)=-u_{10}$. In both cases, the time dependences of $\gamma$, $u_{3}$, and $\phi$ are identical. Nevertheless, since the direction of the magnetic field is reversed, the electron rotates in opposite directions around the $z$ axis in these resonances. Asymptotically, for $l=2$,

$$
\gamma \vec{u}_{\perp} \simeq \frac{\alpha \phi}{\gamma} \hat{\hat{e}}_{1} \simeq-\sqrt{\frac{2 L_{0}}{\gamma}} \hat{\tilde{e}}_{1} .
$$

In this case the electron is accelerated by the circularly polarized component $-\alpha \hat{\tilde{e}}_{2}$ of $\vec{\alpha}$. The accelerating electric fields corresponding to the vector-potential compo- 


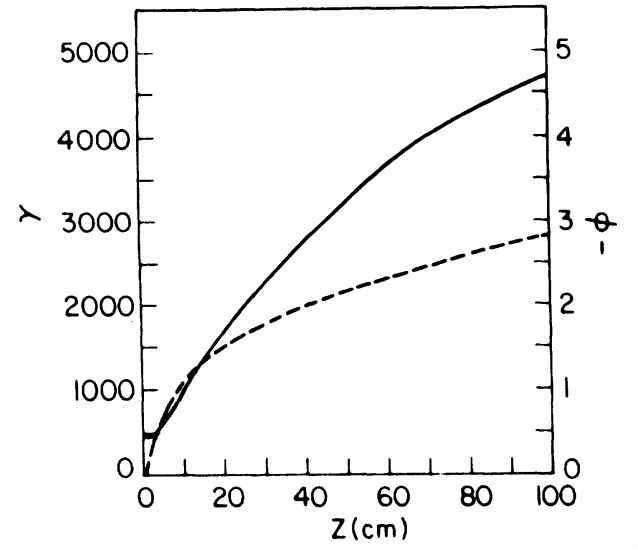

Fig. 1. Autoresonance acceleration of an electron beam with $\gamma_{0}=470$, $u_{10}=u_{20}=0$, by a linearly polarized $\mathrm{Nd}$ : glass radiation (with wavelength $1.06 \mu \mathrm{m}$ ) with $\alpha_{x}=1$ (intensity of $\sim 10^{18} \mathrm{w} / \mathrm{cm}^{2}$ ) along a 100 $\mathrm{kG}$ magnetic field. The $z$ dependence of $\gamma$ (solid line) and $\phi$ (dashed line) is presented.

nents $\left(-\alpha \hat{e}_{2}\right),\left(-\alpha \hat{\tilde{e}}_{2}\right)$ rotate in the directions $\hat{e}_{1}$ and $\hat{\tilde{e}}_{1}$, respectively. Asymptotically, as follows from (32) and (38), the electron rotates in resonance with the electric field associated with the appropriate component of $\vec{\alpha}$, according to the direction of $\vec{\Omega}$. Therefore, asymptotically $(|\phi| \gg>1)$ the acceleration is the same for a linearly polarized radiation with a certain amplitude and a circularly polarized radiation with half of this amplitude. Thus, in accordance with previous calculations [5], the radiation losses in a 1-TeV autoresonance laser accelerator can be negligible. Finally, Fig. 1 shows an example of an autoresonance acceleration by an $\mathrm{Nd}$ : glass laser with $\alpha_{x}=2$, $(\alpha=1$ ), along a 100-kG magnetic field (see (28) and (31)). The $z$ dependence of $\gamma$ and $\phi$ is shown for an electron beam with $\underline{u}_{\perp 0}=0$.

\section{Simultaneous Acceleration of Electrons AND POSITRONS}

The dynamics of a positron in the system are described by (8) and (11) with reversed signs for $\alpha_{1}, \alpha_{2}$, and $\Omega$. For luminous radiation and homogeneous fields, the positron obeys

$$
\left(\gamma u_{1}\right)_{\phi \phi}+l_{p}^{2}\left(\gamma u_{1}\right)=-\left(2+l_{p}\right) \alpha \sin (2 \phi)
$$

where $l_{p}=1-\Omega /\left(k_{0} L_{0}\right)$. Therefore, similarly to an electron, the positron has resonances at $\Omega= \pm k_{0} L_{0}$. After replacing $l$ by $l_{p}$ and changing the sign of $\alpha,(28),(31)$, (35), and (36) describe the nonlinear dynamics of the positron at the resonances $l_{p}=0,2$. At an axial magnetic field $\Omega=k_{0} L_{0}$, the electron and the positron are at the resonances $l=2$ and $l_{p}=0$, respectively, whereas at $\Omega$ $=-k_{0} L_{0}$, the resonances are $l=0$ and $l_{p}=2$. For a given resonant magnetic field the two particles are rotating in opposite directions and are accelerated in phase with different circularly polarized components of $\vec{\alpha}$. Therefore, electrons and positrons can be accelerated simultaneously to high energies. At resonance, the evolution of $\gamma, u_{3}$, and $\phi$ will be the same for electrons and positrons with zero initial transverse velocity $\left(u_{10}=u_{20}=0\right)$. At

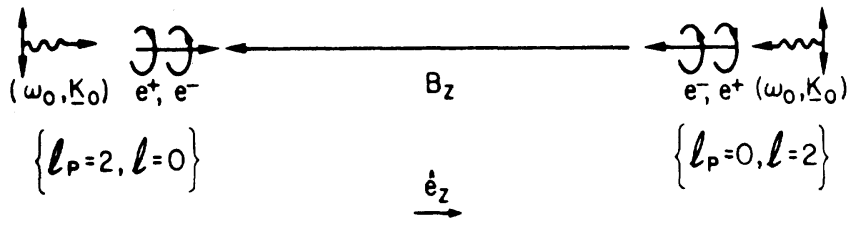

Fig. 2. Autoresonance laser acceleration of oppositely propagating "quasineutral" electron-positron beams along an axial magnetic field $B_{2}$. The types of resonances between the particles and the linearly polarized lasers are indicated.

these conditions, if initially the density of the electrons $N_{e^{-}}(z=0)$ is equal to the density of the positrons $N_{e^{+}}(z$ $=0$ ) in a beam, then

$$
N_{e^{+}}(z)=N_{e^{-}}(z)
$$

for each $z>0$. In conclusion, by using a linearly polarized laser radiation one can accelerate "quasi-neutral" beams (plasmas) containing equal densities of electrons and positrons. Fig. 2 presents a possibility for collinear autoresonance acceleration of "quasi-neutral" electronpositron beams. The beams propagating in the $-z$ and the $+z$ directions are accelerated by the $\left\{l=2, l_{p}=0\right\}$ and the $\left\{l=0, l_{p}=2\right\}$ resonances, respectively.

\section{Discussion and Conclusions}

In this work we have considered the nonlinear electronpositron dynamics in cyclotron autoresonance with a linearly polarized laser radiation propagating along an axial magnetic field. There are two resonances for the electronpositron in the system, $l=2,0\left(l_{p}=0,2\right)$, which differ in the direction of the magnetic field. Accordingly, the particle is accelerated in resonance with one of the circularly polarized components of the laser radiation. Therefore, the acceleration by a linearly polarized radiation of a given amplitude coincides asymptotically with the acceleration by a circularly polarized radiation with half of this amplitude (see (32)-(34) and [5]). It has been shown that simultaneous autoresonance acceleration of electrons and positrons to high energies can be achieved. This result cannot be obtained within the existing schemes for laser acceleration of particles [1], [2]. In other schemes, such as the plasma accelerators [9]-[11] or the inverse free-electron laser [4], a positron is decelerated in a phase at which an electron is accelerated. In contrast, the autoresonance laser acceleration scheme allows continuous acceleration of "quasi-neutral" beams to high energies with low radiation losses. In particular, these beams can contain equal densities of electrons and positrons or other plasmas with oppositely charged particles of equal $|q| / m$ (e.g., positive and negative ions). Such beams can be accelerated without the usual space-charge divergence effects. The acceleration of high-current beams thus seems to be feasible. The guidance of an intense laser beam over large distances is a common problem to all schemes in the far-field approach [1], [2]. There exist several alternatives to the conventional intensity limited waveguides, such as arrays of lenses or plasma fibers [7]. 
The possibility of laser-beam guiding by the electron beams was also discussed recently [12]. Discussion of these methods in application to the ALA scheme presented here is beyond the scope of the present work. Finally, we note that the autoresonance acceleration mechanism might play an important role in cosmic ray acceleration in astrophysical objects, where magnetostatic fields and intense electromagnetic radiation coexist.

\section{REFERENCES}

[1] Laser Acceleration of Particles (AIP Conf. Proc. No. 91), P.J. Channel, Ed. New York: Amer. Inst. Phys., 1982.

[2] The Generation of High Fields for Particles Acceleration to Very High
Energies, Proc. CAS-ECFA-INFN Workshop (Frascati, Italy). Geneva, Switzerland: CERN, 1985.

[3] Laser Acceleration of Particles (AIP Conf. Proc. No. 130), C. Joshi and T. Katsouleas, Eds. New York: Amer. Inst. Phys., 1985.

[4] C. Pellegrini, in AIP Conf. Proc. No. 91. New York: Amer. Inst. Phys., 1982, pp. 138-153.

[5] A. Loeb and L. Friedland, Phys. Rev. A, vol. 33, p. 1828, 1986.

[6] A. Loeb and L. Friedland, submitted to Phys. Lett.

[7] A. Loeb and S. Eliezer, Phys. Rev. Lett., vol. 56, p. 2252, 1986.

[8] K. S. Golovanivsky, IEEE Trans. Plasma Sci., vol. PS-10, no. 3, p. $199,1982$.

[9] T. Tajima and J. M. Dawson, Phys. Rev. Lett., vol. 51, p. 267, 1979.

[10] T. Katsouleas and J. M. Dawson, Phys. Rev. Lett., vol. 51, p. 392, 1982.

[11] P. Chen, J. M. Dawson, R. W. Huff, and T. Katsouleas, Phys. Rev. Lett., vol. 54, p. 693, 1985.

[12] E. T. Scharlemann, A. M. Sessler, and J. S. Wurtele, Phys. Rev. Lett., vol. 54, p. 1925, 1985. 